\title{
Zeitschrift
}

\author{
fur
}

Untersuchung der Nahrungs- und Genußmittel,

sowie der Gebrauchsgegenstände.

\section{Untersuchungen über den Fettgehalt von „Rahmkäsen“".} Von

\author{
K. Windisch, A. Rau, 0. Merger und H. Jesser.
}

Gemeinsame Mitteilungen aus dem Kgl. Technologischen Institut der Landwirtachaftlichen Hochschule Hohenheim, dem Chemischen Laboratorium der Kgl. Zentralstelle fü Gewerbe und Handel in Stuttgart und dem Chemischen Laboratorium der Stadt Stuttgart.

Auf der IX. Hauptversammlung der Freien Vereinigung Deutscher Nahrungsmittelchemiker in Kiel am 17. und 18. Juli 1910 kamen erstmals die Vorschläge des Ausschusses zur Abänderung des Abschnittes „Käse“ zur Besprechung ${ }^{1}$ ). Im Verlauf der Debatte wurde von Prof. Dr. Bömer angeregt, daß man die Grenzzahlen prinzipiell beibehalten, daß man sich aber ihre Höhe erst im nächsten Jahre endgültig schlüssig machen solle. Dr. Grünhut sprach die Ansicht aus, daß man diese Frage nur an Hand von statistischem Material regeln könne, und es liege, wenn man das von Dr. Buttenberg und Dr. Koenig beigebrachte Material ${ }^{2}$ ) betrachte, keine Beobachtung vor, die einen Anlaß biete, den Grenzwert von $45 \%$ bei vollfetten Käsen herabzusetzen. Schließlich gelangte der Antrag von Prof. Dr. Bömer zur Annahme, wonach die Grenzzahlen beibehalten werden und über ihre festzusetzende Höhe im Jahre 1911 Beschlub gefaßt werden sollte. Der Vorsitzende ersuchte dann, die Nahrungsmittelchemiker und die Industrie zur Lösung der Frage bis zum Jahre 1911 beizutragen, und konstatierte noch ausdrücklich, daß die Zahlen noch nicht festgestellt seien und auf Grund derselben eine Verurteilung nicht erfolgen könne. Es wurde dann noch der Beschluß gefaßt, daß man sich mit der Differenzierung von vollfettem, fettem und Magerkäse begnügen und nicht noch weiter zwischen 3/4-fettem und 1/3-fettem Käse unterscheiden wolle. Beschlossen wurde daher, daß es bei folgenden Bestimmungen bleiben soll:

"Bei denjenigen Käsen, bei denen mehrere Fettgehaltsstufen bestehen, müssen diese durch eine entsprechende Bezeichnung, wie Rahmkäse, vollfette, fette, halbfette und Magerkäse bezeichnet werden".

„In neuerer Zeit bürgert sich mehr und mehr der Gebrauch ein, für Sorten- und Marken-

1) Vergl. diese Zeitschrift 1910, 20,376.

2) Diese Zeitschrift 1910, 19, 475.

N. 11. 
käse einen bestimmten Fettgehalt der Trockensubstanz zu garantieren, so wird z. B. der Emmentaler Käse aus einer Milch bereitet, welche auf einen Fettgehalt von $3,1-3,5 \%$ eingestellt ist, sodak der Käse einen Fettgehalt von etwa 48 bis etwa $52 \%$ in der Trockensubstanz hat; ferner wird der Gouda-Käse auf einen Minimalfettgehalt von $45 \%$, der Edamer Käse von $40 \%$ in der Trockensubstanz gearbeitet. In Holland wird jetzt eine staatliche Kontrollmarke auch für Edamer Käse eingeführt, welche einen Fettgebalt von ebenfalls $45 \%$ in der Trockensubstanz garantiert. Die Algäuer Weichkäse, Romadur, Limburger; Weiß̉lacker werden mit einem Feitgehalt der Trockensubstanz von mindestens 30,35 und $40 \%$ hergestellt und in besonderer brauner, bezw. roter oder blauer Umhüllung verkauft."

Dr. Buttenberg und Dr. Koenig legen in ihrer bereits erwähnten Abhandlung bei der Einteilung der Käse folgende Stufen zugrunde:

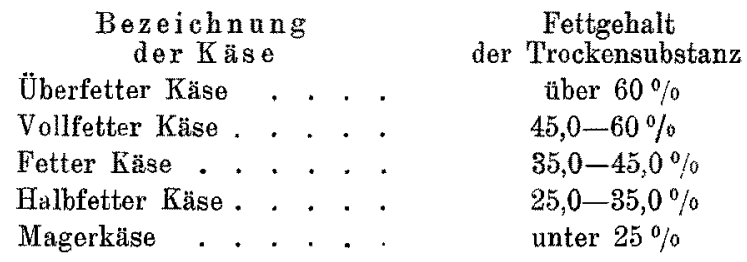

Sie halten sich dabei, abgesehen von dem noch von ihrer Seite besonders beigefügten Wert für überfette Käse, an die von der Deutschen Landwirtschafts-Gesellschaft bei ihren Ausstellungen vorgenommene Einteilung. Um zu zeigen, welchen Fettgehalt man bei einzelnen Käsesorten nach der Literatur erwarten kann, haben Buttenberg und Koenig in der bereits erwähnten Arbeit die diesbezüglichen Angaben zusammengestellt.

Die III. Auflage des „Deutschen Nahrungsmittelbuches“ enthält bezüglich des Fettgehaltes der Käse folgende Bestimmungen:

„1. Rahmkäse und vollfette Käse,

2. Fette Käse.

3. Käse mit einem niedrigeren Fettgehalt und

4. Magerkäse.

Rahmkäse im eigentlichen Sinne sind Käse, welche aus Rahm, oder aus Vollmilch mit einem Zusatz von Rahm bereitet sind, jedach ist es in Deutschland vielfach und seit langer Zeit Gebrauch, auch manche vollfette Käse als Rahmkäse zn bezeichnen.

Vollfette Käse sind aus Vollmilch, bezw. aus Milch mit dem durehschnittlichen Fettgehalt der betr. Viehrasse oder Gegend bereitete Käse. Da dieser Fottgehalt ein recht verschiedener ist, so ist der Fettgehalt vollfetter Käse gleicher Gattung ein verschieden hoher, was selbst noch bei der Angabe des Trockensubstanzfettgehaltes zum Ausdruck kommen dürfte. Ferner sind bei der Beurteilung der Sorten- oder Markenkäse die landesübliehen Bereitungsweisen zu berïcksichtigen.

Als fette Käse sind solche zu bezejchnen, bei deren Bereitung eine höchstens halb entrahmte Milch oder eine Mischung von Vollmilch mit weniger als oder höchstens dem gleichen Teil ganz entrahmeter Milch verwendet ist.

Unter die Käsc mit niedrigerem Fettgehalt sind diejenigen zu zühlen, welche aus mehr als halbentrahmter Milch, oder aus entsprechender Mischung bereitet sind. Im Interesse des reellen Handels liegt es, die Käse niedrigerer Fettgehaltsstufen dureh Bezeichnungen wie $x^{1 / 2-,} 1 / 3-, 1 / 4$-fetter Kasse" kenntlich zu machen.

Magerkäse sind aus Magermilch hergestellte Käse."

Mit Rücksicht auf die bisher bei der Untersuchung von Rahmkäsen in Stutt- 
gart gemachten bereits erwähnten Erfahrungen und im Hinblick auf die in Kiel an die Nahrungsmittelchemiker ergangene Anregung zur Herbeischaffung von statistischem Material hat sich das Chemische Laboratorium der Stadt Stuttgart mit dem Kgl. Technologischen Institut der Landwirtschaftlichen Hochschule in Hohenheim und dem Chemischen Laboratorium der Zentralstelle für Gewerbe und Handel in Stuttgart ins Benehmen gesetzt, um eine größere Serie von als „Rahmkäse“ in Stuttgart verkauften Käse eingehend zu untersuchen. Ein Institut allein wäre neben der Erledigung der laufenden Arbeiten nicht in der Lage gewesen, eine größere Untersuchungsreihe $\mathrm{zu}$ bewältigen und es erschien daher angezeigt, gemeinsam dieses Zahlenmaterial - wie oben angedeutet - zu beschaffen, zumal ja nicht nur die praktische Lebensmittelkontrolle, sondern auch Landwirtschaft und Gewerbe ein berechtigtes Interesse daran haben, auf diesem Gebiet Wandel zu schaffen.

Zur Untersuchung gelangten in den 3 genannten Instituten insgesamt 75 Käse, die in der Zeit von September bis Oktober 1910 in Stuttgart sämtlich als ,Rahmkäse" an den Konsumenten verkauft worden waren. Die einzelnen ermittelten Untersuchungsergebnisse und die auf Grund derselben angestellten Berechnungen sind in der tabellarischen Übersicht enthalten. Die angewendeten Untersuchungsmethoden waren folgende:

\section{Bestiminung des Wassergehaltes.}

Diese erfolgte, einem Vorschlag von Prof. Win disch entsprechend, nach den „Kemptener Vereinbarungen", welche zwischen den Vorständen der milchwirtschaftlichen Versuchsstationen getroffen worden sind.

Das Verfahren ist folgendes:

Man entfernt etwa $1 \mathrm{~cm}$ Außenschicht (Rinde und Schnittfläche), schneidet den Käse in kleine Würfelchen und füllt ihn in Wägegläschen. In einer runden Porzellanschale werden mit einem breiten, hohlen Glaspistill, oder in einer Nickelschale 2-3 g gut zerkleinerter Käse mit etwa $20 \mathrm{~g}$ geglühtem und wieder erkaltetem Seesand versetzt und, nachdem die Schale auf einen Bogen Glanzpapier gestellt ist, Käse und Seesand gründlich vermischt und zerrieben. Alsdann wird etwa eine Stunde lang auf dem Wasserbade und dann im Trockenschrank, am besten im Glycerintrockenschrank bei einer Temperatur von über $100^{\circ}$ noch eine weitere Stunde lang getrocknet. Die Trocknung ist beendigt, wenn 2 gegen Ende der angegebenen Zeit um $1 / 2$ Stunde auseinander liegende Wägungen eine wesentliche, 0,5\% der Trockensubstanz nicht übersteigende Abnahme des Gewichts nicht mehr ergeben. Zu trocknen ist bei Temperaturen von über 100 und bis $115^{\circ}$.

Die Hauptfehler werden bei der Wägung gemacht, da die getrocknete Substanz stark hygroskopisch ist. Man nehme nicht mehr wie $2-3$ g Substanz.

\section{Bestimmung des Fettgehaltes nach Bondzynski-Ratzlaff.}

$2-5 \mathrm{~g}$ der Probe werden in einen runden, mit Kork verschließbaren Stehkolben von etwa $130 \mathrm{ccm}$ Inhalt eingewogen, mit $10 \mathrm{ccm}$ Salzsäure vom spez. Gewicht 1,125 versetzt und auf kleiner Flamme unter Umschwenken vorsiehtig erhitzt. Bei allmählich eintretendem Sieden löst sich der Käse vollständig auf und die Flüssigkeit färbt sich braun. Die etwas abgekühlte Lösung wird noch warm, sodaß das Fett noch flüssig ist, in den Röhrig'schen Apparat (Modifikation des Gotllieb- 
Rös e'schen Apparat) gebracht, mit wenig heißem Wasser nachgespült und die Flüssigkeit im Rohr durch Einstellen in Wasser gekühlt. Darauf wird der im Kölbchen verbliebene Fettrest durch aufeinanderfolgendes, von jedesmaligem kräftigem Umschütteln der Mischungen im Robr unterbrochenes Ausspülen mit $10 \mathrm{cem}$ Alkohol $(95 \%$-ig), $25 \mathrm{ecm}$ Äther und $25 \mathrm{ccm}$ Petroläther in das Rohr gespült, kräftig umgeschüttelt und 3 Stunden absitzen gelassen. Nun läßt man einen aliquoten Teil der Fettlösung durch den Hahn in ein etwa $120 \mathrm{ccm}$ fassendes, gewogenes Erlenmeyer-Kölbchen abfließen, verdunstet das Ätherbenzingemisch bei gelinder Wärme, trocknet das zurückbleibende Fett etwa 2 Stunden bei $100^{\circ}$, läßt im Exsiccator erkalten und wägt den abgewischten Erlenmeyer-Kolben (Vorschlag von Weig mann). Alsdann wird der ermittelte Fettgehalt auf die ganze Fettlösung, entsprechend der angewandten Käsemenge, umgerechnet.

Zur Bestimmung der Refraktion wurde im Städtischen Laboratorium versucht, das Fett aus dem Käse durch Erwärmen auszuschmelzen und abzufiltrieren. Da jedoch, wohl mit Rücksicht auf den geringen Fettgehalt der meisten zur Untersuchung gelangten Käse, bej dieser Methode Schwierigkeiten entstanden, so wurde in der Weise verfahren, daß der Käse mit Bimssteinpulver gut zerrieben wurde, sodaß eine krümelige Masse entstand. Alsdann wurde mit Äther während 48 Stunden durch mehrmaliges Schütteln extrahiert, die abgegossene ätherische Fettlösung mit Chlorcalcium getrocknet, der Äther abdestilliert und das zurückbleibende Fett bei $100^{\circ}$ vollständig vom Äther befreit. Die Refraktometerzahlen der auf diesem Wege erhaltenen Käsefette lagen durchweg etwas niedriger als die bei der Salzsäuremethode (nach B ondzy nsky Ratzlaff) ermittelten Zahlen; letztere sind in der Tabelle jeweils in Klammern gesetzt.

Wie bereits erwähnt, sind sämtliche 75 Käse als „Rahmkäse" an den Konsumenten verkauft worden. Nach den polizeilichen Erhebungen waren von ihnen durch den Fabrikanten bezw. Großhändler 18 als Romadur, 1 als Münsterkäse und 56 als Rahmkäse geliefert worden.

In der tabellarischen Übersicht auf S. 494-495 sind die Käse nach dem Fettgehalt der Trockensubstanz geordnet. Aus dieser Zusammenstellung ergibt sich in Kürze folgendes:

Der Fettgehalt der Trockensubstanz im Käse betrug:

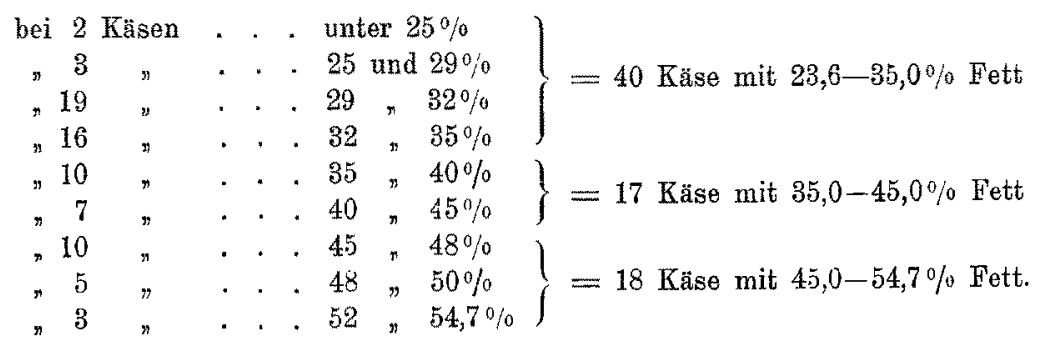

Ordnet man die 75 Käse nach den übrigen analytisch und rechnerisch ermittelten Werten, so ergibt sich folgendes: 
1. Der Wassergehalt betrug:

$$
\begin{aligned}
& \text { bei } 7 \text { Käsen . . . 39,7-50\% } \\
& \text { त24 } n \quad . \quad 50-55 \% \\
& \text { "39 } \quad . .55-60 \% \\
& \Rightarrow 5 * 60-68,1 \% \\
& \text { Gesamt-Schwankungen . . 39,7-63,1\% }
\end{aligned}
$$

2. Der Fettgehalt betrag:

$$
\begin{aligned}
& \text { bei } 1 \text { Käse . . . 8,9\% } \\
& \text { "4: Käsen . . . 11,19-11,44\% } \\
& \text { " } 6 \text { ". . . } 12,32-12,9 \% \\
& " 16, . . . \quad . \quad 13,0-14,0 \% \\
& \text { "14 ". . . . 14,1-14,97\% } \\
& \text { "11 } \quad . . . \quad 15,3-20,1 \% \\
& \text { *20 ". . . . } 20,2-24,6 \% \\
& \because 3 \quad . \quad . \quad . \quad 25,1-27,4 \% \\
& \text { Gesamt-Schwankungen . . } 8,9-27,4^{\circ}
\end{aligned}
$$

3. Die Refraktion des Fettes, in Skalenteilen des Butterrefraktometers ausgedrückt, betrug:

$$
\begin{aligned}
& \text { bei } 8 \text { Käsen . . . } 39,8-48,0 \\
& \text { " } 39 \quad \text {. . . . } 43,1-44,0 \\
& \pi 27 \quad \text {. . . . . } 44,1-44,9 \\
& \text { "1 Kăse . . . . } 46,1 \\
& \text { Gesamt-Sehwankungen . . . } 39,8-46,1
\end{aligned}
$$

4. Der Verkaufspreis betrug:

a) für $100 \mathrm{~g}$ Trockensubstanz

bei 28 Käsen . . 0,217-0,397 M.

n 35 n . . . $0,401-0,495$

.12 $\quad$. . . $0,506-0,599$

Gesamt-Schwankungen . 0,217-0,599 M. b) für $100 \mathrm{~g} \mathrm{Fett}$ bei 20 Käsen . . 0,568-0,883 M.

$$
\begin{aligned}
& \text { " } 17 . \quad \text {. . . 0,901-1,182 } \\
& , 30, \quad . \quad 1,247-1,687
\end{aligned}
$$

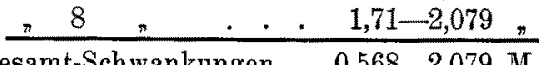

5. Für 1 Mk. erbält man Fett im Käse:

$$
\begin{aligned}
& \text { bei } 11 \text { Käson . . . . 48,1- } 59,8 \mathrm{~g} \\
& \text { n } 12 \text {. . . . } 60,6-69,5 \mathrm{~g} \\
& \text { "14 . . . } 70,4-79,0 \mathrm{~g} \\
& \text { "7 } 7 . . .80,1-89,6 \mathrm{~g} \\
& \text { " } 9 \text {. . . . } 95,0-108,3 \mathrm{~g} \\
& \text { "8 . . . . 106,3-119,0 g } \\
& \text { " } 9 \text {. . . . 120,3-140,5 g } \\
& \text { " } 4 \text {. . . . 145,4-155,05 g } \\
& " 1 \% \text {. . . } 176,0 \mathrm{~g} \\
& \text { Gesamt-Schwankungen . . } 48,1-176,0 \mathrm{~g}
\end{aligned}
$$

6. Das Verhältnis von Fett zur fettfreien Trockensubstanz im Käse war: bei 9 Käsen . . . 1:0,82-1,09

$$
\begin{aligned}
& , 31, \text {. . . . 1:1,10-1,98 } \\
& \text { n33 } n \text {. . . . } 1: 2,01-2,86 \\
& \pi 2, \quad \text {. . . . } 1: 3,21-3,24
\end{aligned}
$$


Untersuchungsergebnisse.

\begin{tabular}{|c|c|c|c|c|c|c|c|c|c|c|c|c|c|c|c|}
\hline \multirow{4}{*}{$\dot{0}$} & \multicolumn{9}{|c|}{ Ergebnisse der chemischen Untersuebung } & \multirow{4}{*}{ 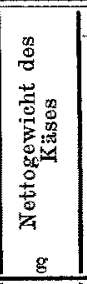 } & \multicolumn{5}{|c|}{ Preisverhältnisse } \\
\hline & \multirow{3}{*}{ 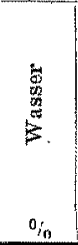 } & \multirow{3}{*}{ Fett } & \multirow{3}{*}{ 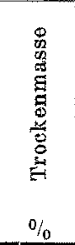 } & \multirow{3}{*}{ 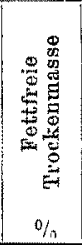 } & \multicolumn{3}{|c|}{$\begin{array}{c}\begin{array}{c}\text { Besondere Prüfung } \\
\text { des Fettes }\end{array} \\
\end{array}$} & \multirow{3}{*}{ 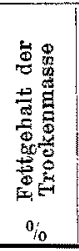 } & \multirow{3}{*}{ 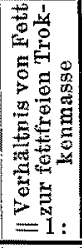 } & & \multirow{3}{*}{ 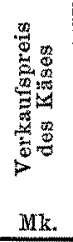 } & \multicolumn{3}{|c|}{ Preis für : } & \multirow{3}{*}{ 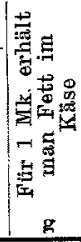 } \\
\hline & & & & & \multirow[b]{2}{*}{ 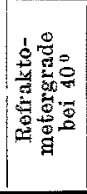 } & \multicolumn{2}{|c|}{$\begin{array}{l}\text { Sesamöl- } \\
\text { Realktion } \\
\end{array}$} & & & & & & & & \\
\hline & & & & & & 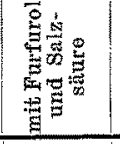 & 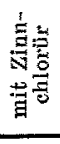 & & & & & $\begin{array}{l}100 \mathrm{~g} \\
\text { Käse }\end{array}$ & 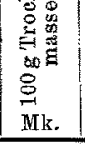 & $\begin{array}{c}100 \mathrm{~g} \\
\text { Fett } \\
\text { Mk. }\end{array}$ & \\
\hline 1 & 39,7 & 14,23 & 60,3 & 46,07 & 44,6 & 0 & 0 & 23,6 & 3,24 & 92 & 0,20 & 0,217 & 0,361 & 1.527 & 65,5 \\
\hline 2 & 62,5 & 8,9 & 37,5 & 28,6 & 46,1 & 0 & 0 & 23,7 & 3,21 & 54,2 & 0,10 & 0,185 & 0,493 & 2,079 & 48,1 \\
\hline 3 & 50.8 & 11,44 & 44,2 & 32,76 & 44,6 & 0 & 0 & 25,88 & 2,86 & 95 & 0,20 & 0.211 & 0,476 & 1,840 & 54,5 \\
\hline 4 & 58,5 & 11,4 & 41,5 & 30,1 & 44,2 & 0 & 0 & 27,5 & 2,64 & 104 & 0,20 & 0,192 & $0,463 \mid$ & 1,687 & 59,3 \\
\hline 5 & 55,7 & 12,7 & 44,3 & 31,6 & 43,1 & 0 & 0 & 28,67 & 2,49 & 100,0 & 0,20 & 0,200 & 0,451 & 1,570 & 63,7 \\
\hline 6 & 57,0 & 12,9 & 43,0 & 30,1 & 42,8 & 0 & 0 & 30,0 & 2,33 & 90,6 & 0,15 & 0,166 & 0,386 & 1,287 & 77,7 \\
\hline 7 & 58,7 & 12,4 & 41,3 & 28,9 & 44,0 & 0 & 0 & 30,0 & 2,33 & 86 & 0,18 & 0,209 & 0,506 & 1,685 & 59,3 \\
\hline 8 & 54,16 & 13,88 & 45,84 & 32,01 & $\begin{array}{l}44,0 \\
(45,0)\end{array}$ & 0 & 0 & 30,17 & 2,30 & 100,0 & 0,20 & 0,200 & 0,436 & 1,450 & 68,9 \\
\hline 9 & 55,8 & 13,5 & 44,7 & 31,2 & 43,1 & 0 & 0 & 30,2 & 2,31 & 97,8 & 0,20 & 0,205 & 0,459 & 1,519 & 65,8 \\
\hline 10 & 63,1 & 11,19 & 36,9 & 25,71 & 41 & 0 & 0 & 30,32 & 2,30 & 90,5 & 0,20 & 0,221 & 0,599 & 1,975 & 50,7 \\
\hline 11 & 52,51 & 14,42 & 47,49 & 33,07 & $\begin{array}{c}44,9 \\
(46,3)\end{array}$ & 0 & 0 & 30,37 & 2,30 & 83,0 & 0,20 & 0,241 & 0,507 & 1,671 & 59,8 \\
\hline 12 & 62,8 . & 11,3 & 37,2 & 25,9 & 44,0 & 0 & 0 & 30,4 & 2,29 & 103 & 0,20 & 0,199 & 0,522 & 1,718 & 58,2 \\
\hline 13 & 52,24 & 14,53 & 47,76 & 33,23 & $\begin{array}{l}42,8 \\
(44,9)\end{array}$ & - & - & 30,42 & 2,29 & 95,0 & 0,20 & 0,210 & 0,439 & 1,449 & 69,0 \\
\hline 14 & 57,0 & 13,1 & 43,0 & 29,9 & 43,3 & 0 & 0 & 30,5 & 2,28 & 106 & 0,20 & 0,190 & 0,438 & 1,440 & 69,4 \\
\hline 15 & 60,2 & 12,32 & 39,8 & 27,48 & 43,6 & 0 & 0 & 30,96 & 2,24 & 85,5 & 0,18 & 0,210 & 0,529 & 1,710 & 58,5 \\
\hline 16 & 58,2 & 13,1 & 41,8 & 28,7 & 43,3 & 0 & 0 & 31,3 & 2,19 & 84 & 0,20 & 0,238 & 0,569 & 1,817 & 55,0 \\
\hline 17 & 60,4 & 12,4 & 39,6 & 27,2 & 44,5 & 0 & 0 & 31,3 & 2,19 & 99 & 0,20 & 0,202 & 0,510 & 1,629 & 61,4 \\
\hline 18 & 59,4 & 12,74 & 40,6 & 27,86 & 44,1 & 0 & 0 & 31,38 & 2,19 & 124 & 0,20 & 0,169 & 0,397 & 1,266 & 79,0 \\
\hline 19 & 59,0 & 13,0 & 41,0 & 28,0 & 43,6 & 0 & 0 & 31,70 & 2,15 & 100,0 & 0,18 & 0,180 & 0,439 & 1,380 & 72,2 \\
\hline 20 & 58,3 & 13,2 & 41,7 & 28,5 & 43,4 & 0 & 0 & 31,7 & 2,16 & 115,7 & 0,18 & 0,156 & 0,374 & 1,182 & 84,6 \\
\hline 21 & 56,84 & 13,70 & 43,16 & 29,46 & 44,2 & - & - & 31,74 & 2,15 & 110,0 & 0,20 & 0,181 & 0,421 & 1,320 & 75,8 \\
\hline 22 & 59,01 & 13,05 & 40,99 & 27,94 & $\left(\begin{array}{l}44,0 \\
(45,0)\end{array}\right.$ & - & 一 & $31,83 \mid$ & 2,14 & 105,0 & 0,20 & 0,190 & 0,465 & 1,460 & 68,5 \\
\hline 28 & 59,3 & 13,0 & 40,7 & 27,7 & 43,8 & - & - & 31,9 & 2,13 & 99 & 0,17 & 0,172 & 0,418 & 1,308 & 76,5 \\
\hline 24 & 56,5 & 13,9 & 43,5 & 29,6 & 43,7 & 0 & 0 & 31,9 & 2,13 & 100 & 0,20 & 0,200 & 0,460 & 1,440 & 69,5 \\
\hline 25 & 55,0 & 14,5 & 45,0 & 30,5 & 44,1 & 0 & 0 & 32,2 & 2,10 & 97,2 & 0,20 & 0,206 & 0,458 & 1,421 & 70,4 \\
\hline 26 & 59,5 & 13,1 & 40,5 & 27,4 & 44,0 & 0 & 0 & 39,4 & 2,09 & 86,8 & 0,20 & 0,232 & 0,578 & 1,770 & 56,5 \\
\hline 27 & 57,79 & 13,75 & 42,21 & 28,46 & $\begin{array}{l}44,3 \\
(46,0)\end{array}$ & 0 & 0 & 32,57 & 2,07 & 100,0 & 0,18 & 0,180 & 0,426 & 1,309 & 76,4 \\
\hline 28 & 56,0 & 14,4 & 44,0 & 29,6 & 42,8 & $\begin{array}{l}\text { sehwache } \\
\text { Rotfärbg. }\end{array}$ & 0 & 32,7 & 2,06 & 96,8 & 0,16 & 0,165 & 0,375 & 1,146 & 87,3 \\
\hline 29 & 59,08 & 13,38 & 40,92 & 27,54 & $\begin{array}{l}44,7 \\
45,3)\end{array}$ & 0 & 0 & 32,7 & 2,06 & 105,0 & 0,20 & 0,190 & 0,465 & 1,420 & 70,4 \\
\hline 30 & 57,4 & 14,0 & 42,6 & 28,6 & 44,5 & 0 & 0 & 32,9 & 2,04 & 97 & 0,20 & 0,206 & 0,484 & 1,471 & 68,0 \\
\hline 31 & 59,18 & 13,46 & 40,82 & 27,36 & $\begin{array}{l}44,9 \\
(46,6)\end{array}$ & - & - & 32,97 & 2,03 & 90 & 0,20 & 0,222 & 0,546 & 1,651 & 60,6 \\
\hline 32 & 56,7 & 14,3 & 43,3 & 29,0 & 43,9 & 0 & 0 & 33,02 & 2,03 & 100,0 & 0,20 & 0,200 & 0,462 & 1,400 & 71,5 \\
\hline 33 & 56,5 & 14,4 & 43,5 & 29,1 & 44 & 0 & 0 & 33,1 & 2,02 & 143 & 0,20 & 0,140 & 0,322 & 0,972 & 102,9 \\
\hline 34 & 58,3 & 13,84 & $=41,7$ & 27,86 & \begin{tabular}{|l|l}
5 & 44,8
\end{tabular} & 0 & 0 & 33,19 & 2,01 & 83 & 0,20 & 0,241 & 0,578 & 1,740 & 57,5 \\
\hline 35 & 56,0 & 14,6 & 44,0 & 29,4 & $4 \hat{4} .9$ & 0 & 0 & 33,2 & 2,01 & 158 & 0,20 & 0,127 & 0,288 & 0,870 & 114,9 \\
\hline 36 & 56,86 & 14,46 & 43,14 & 28,68 & $(43,6)$ & 0 & 0 & 33,52 & 1,98 & 100,0 & 0,20 & 0,200 & 0,468 & 1,389 & 72,3 \\
\hline 37 & 58,0 & 14,1 & 42,0 & 27,9 & 44,0 & 0 & 0 & 38,6 & 1,98 & 94,1 & 0,18 & 0,191 & 0,455 & 1,355 & 73,8 \\
\hline
\end{tabular}




\begin{tabular}{|c|c|c|c|c|c|c|c|c|c|c|c|c|c|c|c|}
\hline \multirow{4}{*}{$\dot{0}$} & \multirow[b]{4}{*}{ 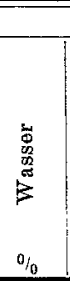 } & \multicolumn{8}{|c|}{ Ergebnisse der chemischen Ũtersuchung } & \multirow{4}{*}{ 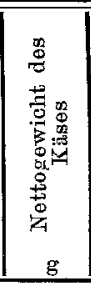 } & \multicolumn{5}{|c|}{ Preisverhältnisse } \\
\hline & & \multirow{3}{*}{ Fett } & \multirow{3}{*}{ 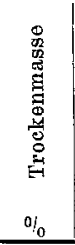 } & \multirow{3}{*}{ 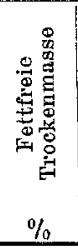 } & \multicolumn{3}{|c|}{$\begin{array}{c}\text { Besondere Prüfung } \\
\text { des Fettes }\end{array}$} & \multirow{3}{*}{ 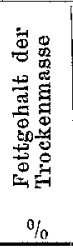 } & \multirow{3}{*}{ 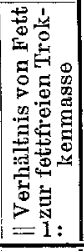 } & & \multirow{3}{*}{ 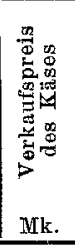 } & \multicolumn{3}{|c|}{ Preis für : } & \multirow{3}{*}{ 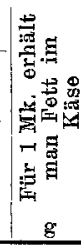 } \\
\hline & & & & & \multirow[b]{2}{*}{ 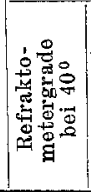 } & \multicolumn{2}{|c|}{$\begin{array}{l}\text { Sesamö̈l- } \\
\text { Reaktion } \\
\end{array}$} & & & & & & & & \\
\hline & & & & & & 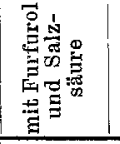 & 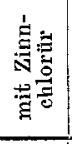 & & & & & $\begin{array}{l}100 \mathrm{~g} \\
\text { Käse }\end{array}$ & 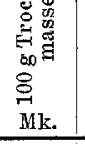 & $\begin{array}{l}100 \mathrm{~g} \\
\text { Fett }\end{array}$ & \\
\hline 38 & 57,2 & 14,5 & 42,8 & 28,3 & 44,1 & 0 & 0 & 33,87 & 1,95 & 100,0 & 0,20 & 0,200 & 0,468 & 1,38 & 72,5 \\
\hline 39 &, 76 & 4,92 & 43,24 & 28,32 & 44,0 & 0 & 0 & 34,5 & 1,90 & 95,0 & 0,20 & 0,210 & 0,486 & 1,41 & 70,87 \\
\hline 40 &, 4 & 14,8 & 42,6 & 27,8 & 39,8 & 0 & 0 & 34,7 & 1,88 & 107 & 0,18 & 0,168 & 0,394 & 1,135 & 88,1 \\
\hline 41 & 53,8 & 16,2 & 46,2 & 30,0 & 43,0 & 0 & 0 & 35,1 & 1,85 & 106,5 & 0,18 & 0,169 & $0,3 \in 6$ & 1,043 & 95,9 \\
\hline 42 & 57,41 & 97 & 42,59 & 27,62 & $\begin{array}{l}43,4 \\
(44,2)\end{array}$ & 0 & 0 & 35,14 & & 105,0 & 0,20 & 0,190 & 0,447 & 1,272 & 78,59 \\
\hline 43 & 6,6 & 15,3 & 43,4 & 28,1 & 44,1 & 0 & 0 & 35,3 & 1,84 & 129 & 0,20 & 0,155 & 0,357 & 1,013 & 98,7 \\
\hline 44 & 4,4 & 16,7 & 45,6 & 28,9 & 43,6 & 0 & 0 & 36,6 & 1,73 & 83 & 0,20 & 0,241 & 0,529 & 1,443 & 69,3 \\
\hline 45 & 5,64 & 20,2 & 54,36 & 34,16 & 48,9 & 0 & 0 & 37,16 & 1,69 & 105,0 & 0,18 & $0,171 \mid$ & 0,315 & 0,848 & 3118,0 \\
\hline 46 & 0,28 & 19,08 & 49,72 & 30,64 & $=44,7$ & 0 & 0 & 38,37 & 1,61 & 105,0 & 0,25 & 0,238 & 0,479 & 1,247 & 80,12 \\
\hline 47 & 6,3 & 16,9 & 43,7 & 26,8 & 44,7 & 0 & 0 & 38,67 & & 106 & 0,20 & 0,189 & 0,432 & 1,116 & \begin{tabular}{|l|l}
69,6 \\
\end{tabular} \\
\hline 48 &, 5 & 18,17 & 46,5 & 28,33 & 44,8 & 0 & 0 & 39,07 & & 117 & 0,20 & 0,171 & 0,368 &, 941 & 106,3 \\
\hline 49 & 56,32 & 17,15 & 43,68 & 26,53 & 43,6 & 0 & 0 & 39,26 & 54 & 100,0 & 0,20 & $|0,200|$ & 0,458 & 1,166 & 3) 85.7 \\
\hline 50 & 8,8 & 17,1 & 43,2 & 26,1 & 41,0 & 0 & 0 & 39,6 & 1,53 & 132,3 & 0,20 & & 0,350 & 0,883 & 113,2 \\
\hline 51 & 42,39 & 23,09 & 57,61 & 34,52 & 43,7 & 0 & 0 & 40,08 & 1,49 & 120,0 & 0,18 & 0,150 & 0,260 & 0,649 & 154,1 \\
\hline 52 & 56,76 & 17,61 & 43,24 & 25,63 & $\begin{array}{l}43.9 \\
(44,6)\end{array}$ & 0 & 0 & 40,72 & & 110,0 & 0,15 & & 0.315 & & \\
\hline 53 & 4,73 & 26 & 55,27 & 32,01 & 44,7 & 0 & 0 & & 1,38 & & 0,18 & 150 & 271 & 645 & 155,0 \\
\hline 54 & 54,16 & 19,76 & 45,84 & 26,08 & $\begin{array}{c}44,4 \\
(45,6)\end{array}$ & 0 & 0 & 43,11 & 1,32 & 120,0 & 0,20 & 0,166 & 0,363 & 0,84 & 119,0 \\
\hline 55 & 50,07 & 22,11 & 49,93 & 27,82 & 43,9 & 0 & 0 & 4,82 & 1,25 & 110,0 & 0,20 & 182 & 0,364 & 0,822 & 2121,6 \\
\hline 56 & 4,6 & 20,1 & 45,4 & 25,3 & 44, & 0 & 0 & & 1,26 & 98 & 0,20 & 0,204 & 0,445 & 1,015 & $5 \quad 98,5$ \\
\hline 57 & 3,7 & 20,65 & 46,30 & 25,65 & 43,9 & 0 & 0 & 44,6 & 1,24 & 100,0 & 0,20 & 0,200 & 0,432 & 0,96 & 8103 \\
\hline 58 & 53,66 & 20,92 & 46,34 & 25,42 & 44,3 & 0 & 0 & 45,14 & 1,22 & 115,0 & 0,20 & 0,174 & 0,375 & 0,831 & 1120 \\
\hline 59 & 50,8 & $22,3 \mid$ & 49,2 & 26,9 & 43,4 & 0 & 0 & 45,3 & 1,21 & 95 & 0,18 & 0,189 & 0,384 & 0,848 & 117 \\
\hline 60 & 54,6 & 20,7 & 45,4 & 24,7 & 44 & 0 & 0 & 45,6 & 1,19 & 97 & 0,20 & 0,206 & 0,444 & 0,996 & 100 \\
\hline 61 & 51,2 & 22,3 & 48,8 & 26,5 & 43,1 & $\begin{array}{l}\overline{\text { sehwac }} \\
\text { färl }\end{array}$ & & 45,7 & 1,19 & 99,7 & 0,20 & 0,201 & 0,412 & 0,901 & 111, \\
\hline 62 & 49,12 & 23,31 & 50,88 & 27,57 & $\begin{array}{l}44,0 \\
(44,9)\end{array}$ & $\begin{array}{l}\operatorname{rar} \\
0\end{array}$ & 0 & 45,81 & 1,18 & 110,0 & 0,20 & 0,181 & 0,357 & 0,78 & 100 \\
\hline 63 & & & & 25 & 43,3 & 0 & 0 ! & 47,01 & & 100,0 & & 240 & 195 & & 5 , \\
\hline 64 & 5,0 & 21,3 & 45,0 & 23,7 & 43 & c & 0 & 47,3 & 1,11 & 81,5 & 0,20 & 0,245 & 0,544 & $1,1 \leq 0$ & 0.87 , \\
\hline 65 & 50,0 & 23,74 & & 26,26 & 44 & 0 & 0 & 47,48 & 1,11 & 84 & 0,15 & 0,179 & 0,358 & 0,754 & 4132 \\
\hline 66 & 51,02 & 23,31 & 48,98 & 25,67 & $\begin{array}{l}43, \\
44,\end{array}$ & 0 & 0 & 47,59 & 1,10 & 120,0 & 0,20 & 0,166 & 0,340 & 0,712 & 2140 \\
\hline 67 & 54,87 & $21, \overline{5} 3$ & 45,13 & 23,6 & $\begin{array}{l}43 \\
(44\end{array}$ & 0 & 0 & 47,7 & 1,09 & 95,0 & 0,20 & 0,211 & 0,467 & 0,978 & \begin{tabular}{l|l}
8 & 120
\end{tabular} \\
\hline 68 & 2,4 & & 47,6 & 24,4 & 43, & 0 & 0 & & 105 & 94 & 0,15 &, 159 & & 0,688 & $8 \mid 145$ \\
\hline 69 & 48,5 & 25,1 & 51,5 & 26,4 & 44,7 & 0 & 0 & 48,7 & 1,05 & 95,8 & 0,18 & 0,188 & 0,365 & 0,749 & 9133, \\
\hline 70 & 51,6 & 23,6 & 48,4 & 24,8 & 43,5 & & 0 & 48,8 & 1,05 & 123,6 & 0,24 & 0,194 & 0,401 & 0,822 & 2121, \\
\hline 71 & 50,5 & & & 24,9 & 43 & 0 & c & & & 2,2 & 0,20 & 0,178 & 0,360 & 0,724 & 4138 \\
\hline 62 & 52,1 & 23, & 47,9 & 24,0 & 43, & 0 & 0 & & 1,00 & 1 & 0,25 & 236 &, 493 & 0,987 & $\begin{array}{lll}7 & 101\end{array}$ \\
\hline 73 & 56,9 & 22,4 & 43,1 & 20,7 & 44 & 0 & 0 & & 0,8 & 131,2 & 0,25 & 0,191 & 0,443 & 0,853 & 3117 \\
\hline 74 & 49,4 & 27,4 & 50,6 & 23,2 & 44,3 & 0 & 0 & 54,1 & 0,85 & 9,4 & 0,20 & 0,183 & 0,362 & 0,668 & 8149 \\
\hline 75 & 54,01 & 25,17 & 45,99 & 20,82 & $\mid(46,6)$ & 0 & 0 & 54,73 & 0,82 & 105,0 & 0,15 & 0,143 & 0,311 & 0,568 & 8176 , \\
\hline
\end{tabular}


Wenn man die in der Tabelle enthaltenen Untersuchungsergebnisse übersieht, so ist aus ihnen die bedauerliche Tatsache zu entnehmen, dass ein sehr großer Prozentsatz der durchweg vom Detailisten dem Konsumenten und vielfach auch vom Fabrikanten oder Groluändler dem Detailisten als „Rahmkäse" verkauften Produkte sehr fettarm war. $53,3 \%$ der untersuchten Käse zeigten einen Fettgehalt von $35 \%$ und darunter in der Trockenmasse, $22,7 \%$ einen solchen von $35-45 \%$ und $24 \%$ einen solchen von $45-54,7 \%$.

Nach den eingangs erwähnten Abänderungsvorschlägen würde kein einziger der untersuchten Käse als "Rahmkäse" angesehen werden können, da der höchste beobachtete Fettgehalt immer noch etwas unter der festgesetzten Mindestgrenze für „Rahmkäse" von $55 \%$ liegt. Als Fettkäse mit mindestens $30 \%$ Fettgehalt dagegen hätten etwa $92 \%$ der untersuchten Käse gelten können. Man wird sich nach den Untersuchungsergebnissen nicht verhehlen können, daß sehr große Unterschiede hinsichtlich des wirklichen Wertes der verschiedenen Käse in die Erscheinung treten, sodaß auch die reellen Fabrikanten allen Anlaß haben, auf die Schaffung geordneter Verhältnisse hinzuarbeiten, andererseits wird man sich aber auch der Einsicht nicht verschließen können, daß hier in Stuttgart und in Württemberg wohl allgemein das konsumierende Publikum seit langem „Rahmkäse“ kauft, die keine eigentlichen Rahmkäse, sondern in der Regel nicht einmal vollfette, sondern meist nur halbfette Käse sind.

\title{
Über einen vereinfachten Flnor-Nachweis.
}

\author{
Von \\ E. Rupp in Königsberg. \\ [Eingegangen am 8. September 1911.]
}

Der Nachweis von Fluoriden gemäß den Ausführungsbestimmungen ${ }^{1}$ ) zu dem Gesetze, betreffend die Schlachtvieh- und Fleischbeschau vom 3. Juni 1900 läßt an Empfindlichkeit nichts zu wünschen übrig, vorausgesetzt, daß das Asche-Schwefelsäuregemisch hinlänglich erhitzt und die Deckschale genügend gekühlt wird, um ein Ausfließen der Wachsmarkierungen zu verhüten. Zu letzterem Zwecke ist auf die Verwendung eines Stückchens Eis verwiesen. Wo solches nicht zur Stelle ist, kann man sich, wie von Treadwell u. a. beschrieben, mit der Kühlwirkung eines von Wasser durchsickerten Baumwollfadens behelfen.

Da dies immerhin etwas umständlich ist und auch das Bewachsen der Uhrschalen Zeitaufwand erfordert, sei hiermit ein Verfahren beschrieben, welches beides überflüssig macht.

Es wird dabei die Glasätzwirkung des Fluorwasserstoffs mit der durch Siliciumfluorid hervorbringbaren Trübung eines Wassertropfens verknüpft.

$$
\begin{gathered}
\mathrm{Na}_{2} \mathrm{Ca} \mathrm{Si} \mathrm{O}_{64}+28 \mathrm{HF}=14 \mathrm{H}_{2} \mathrm{O}+\mathrm{Na}_{2} \mathrm{SiF}_{6}+\mathrm{CaSiF}_{6}+4 \mathrm{SiF}_{4}, \\
\text { (Natronglas) } \\
3 \mathrm{SiF}_{4}+3 \mathrm{H}_{2} \mathrm{O}=2 \mathrm{H}_{2} \mathrm{SiF}_{6}+\mathrm{H}_{2} \mathrm{SiO}_{3} .
\end{gathered}
$$

1) Diese Zeitschrift $1908,15,565$. 